\title{
Sorafenib versus sunitinib as first-line treatment agents in Chinese patients with metastatic renal cell carcinoma: the largest multicenter retrospective analysis of survival and prognostic factors
}

\author{
Hai-Liang Zhang ${ }^{1,2+}$, Xi-Nan Sheng ${ }^{3+}$, Xue-Song Li ${ }^{4}$, Hong-Kai Wang ${ }^{1,2}$, Zhi-Hong Chi ${ }^{3}$, Zhi-Song He ${ }^{4}$,
} Ding-Wei Ye $\mathrm{P}^{12^{*}}$ and Jun Guo ${ }^{3^{*}}$

\begin{abstract}
Background: To compare the efficacy of sorafenib and sunitinib with regard to overall survival (OS) and progression free survival (PFS) in Chinese patients with metastatic renal cell carcinoma (mRCC).

Methods: A multicenter, retrospective study was performed to elucidate the relationship between clinical variables and prognosis comparing sorafenib and sunitinib as first-line treatment agents in Chinese patients with mRCC. Between September 2006 and December 2014, 845 patients received either sorafenib (400 mg bid; $n=483$ ) or sunitinib (50 mg q.d; $n=362$ ). The primary end point was OS and PFS.

Results: The percentage of patients with low and moderate risk according to Memorial Sloan-Kettering Cancer Centre (MSKCC) score was significantly higher in sunitinib group, and that with high risk was significantly higher in sorafenib group (15.1 vs. 5.2\%; $p<0.001$ ). Median OS was similar in sorafenib and sunitinib group (24 vs. 24 months; $p=0.298$ ). Sorafenib group exhibited higher mPFS compared to sunitinib group (11.1 vs. 10.0 months; $p=0.028$ ). Treatment (sorafenib vs sunitinib), pathology, Eastern Cooperative Oncology Group (ECOG) performance status, MSKCC scores, Heng's criteria of risk, and number of metastases were identified as significant predictors for OS and along with liver metastasis for PFS. Clinical outcomes in terms of mOS was significantly better with sorafenib in patients $\geq 65$ years of age $(p=.041)$, ECOG $0(p=0.0001)$, and median MSKCC risk score $(p=0.008)$.
\end{abstract}

Conclusions: Sorafenib and sunitinib are both effective in treating mRCC. However, sorafenib might be more effective in elderly patients ( $\geq 65$ years) and in patients with an ECOG status of 0, classified under MSKCC moderate risk.

Keywords: Metastatic renal cell carcinoma, Sorafenib, Sunitinib, Prognosis, Survival

\footnotetext{
*Correspondence: dwyeli@163.com; guoj307@126.com

Hai-Liang Zhang and Xi-Nan Sheng are co-first authors.

${ }^{\dagger}$ Equal contributors

'Department of Urology, Fudan University Shanghai Cancer Center, Shanghai 200032, People's Republic of China

${ }^{3}$ Key Laboratory of Carcinogenesis and Translational Research (Ministry of Education/Beijing), Department of Renal Cancer and Melanoma, Peking University Cancer Hospital \& Institute, Beijing, People's Republic of China Full list of author information is available at the end of the article
} 


\section{Background}

It is evident that $\sim 30 \%$ of renal cell carcinoma (RCC) patients have overt metastases, defined as metastatic RCC (mRCC) [1] with a very poor average 5-year survival rate (only 10-12\%) [2]. The growing evidence on the associations of molecular mechanisms with $\mathrm{mRCC}$ and also the abstinence of cytokine-based therapies due to high toxicity profile [3-5] has rationalized several randomized clinical trials on molecular targeted therapies such as sorafenib [6], bevacizumab [4], temsirolimus [7], sunitinib [8], pazopanib [9], everolimus [10], and axitinib [11] as first- and second-line treatment, which were found to be efficacious and safer than conventional immunotherapy. The availability of these targeted therapies has resulted in prolonged overall survival (OS) to approximately 2 years, thereby emerging as the standard of care in the management of mRCC. However, efficacy of drugs used in cancer chemotherapy is often associated with distinctive challenges due to infrequent occurrence of measurable disease, prolonged natural history of disease, diverse clinical characteristics and greater likelihood of contrary outcomes when treating elderly patients with more aggressive treatments $[12,13]$. These challenges also influence regular, as well as accelerated regulatory approvals of drugs, which require extensive evidence of efficacy derived from clinical trials in addition to accommodating integral characteristics of disease and patient population [14]. Also, first-line therapies should be strategically chosen in order to devoid the need of sequential therapy with second-line therapies. For this purpose, comparing the efficacy of drugs may offer substantial evidence and guidance on the optimal use of targeted therapies. When evaluated as first-line treatment, axitinib demonstrated clinical efficacy and safety, but no significant progression free survival (PFS) benefit over sorafenib in a Phase III randomized comparison [15]. Further, sunitinib had similar efficacy as pazopanib in a non-inferiority trial [16]. Moreover, in case of sunitinib failure in advanced RCC, everolimus and axitinib appear to provide second-line PFS benefits [17]. On the other hand, recent Phase III Investigating Torisel as Second-Line Therapy (INTORSECT) trial reported no significant benefit of either temsirolimus or sorafenib as second-line treatment after sunitinib failure [7], though, temsirolimus demonstrated clinical efficacy as first-line therapy in poor risk patients [18]. Although sorafenib is a comparator agent in several clinical trials and often used as a second-line therapy, Chinese patients have been more responsive to sorafenib than western patients, hence, both sunitinib and sorafenib are widely recommended first-line therapies in China [19]. However, studies directly comparing efficacy of the two therapies in first-line settings which may guide the clinical decisions of mRCC treatment in Asian patients are limited. Although, a Korean study has reported comparable efficacy of the 2 drugs in mRCC patients, the findings were limited due to small patient population and a single centric retrospective design, warranting additional investigation [20]. Hence this study aims to retrospectively elucidate the relationship between clinical variables and disease prognosis by comparing sorafenib versus sunitinib in Chinese patients with $\mathrm{mRCC}$ at 3 tertiary hospitals in China.

\section{Methods \\ Patient population}

Records of patients with mRCC were maintained at the Beijing Cancer Hospital, Fudan University Shanghai Cancer Centre and the Peking University First Hospital. Between September 2006 and December 2014, the patient records were retrospectively reviewed and computed tomography (CT) scans were independently reviewed by a senior radiologist, blinded to a treatment arm.

Patients between 18 and 84 years of age; histological confirmation of advanced/mRCC; unsuitable for cytokine therapy; no prior systemic therapy; Eastern Cooperative Oncology Group performance status (ECOG PS) 0 to 3; 1 or more measurable lesions by $\mathrm{CT}$ or magnetic resonance imaging (MRI) according to Response Evaluation Criteria in Solid Tumors (RECIST 1.0); favorable or intermediate Memorial Sloan Kettering Cancer Centre (MSKCC) risk score; adequate bone marrow, liver, and renal function and willing to undergo first-line targeted therapy with sorafenib or sunitinib are included. Patients were excluded if they had unstable or severe cardiac disease; active, clinically serious infection or symptomatic metastatic brain tumor and with ECOG PS 4 and 5. Ethical approval was obtained from institutional ethics committee of Beijing Cancer Hospital, Fudan University Shanghai Cancer Centre and the Peking University First Hospital, and the protocol conformed to the principles of declaration of Helsinki, its subsequent revisions. Patient signed informed consent was obtained.

\section{Treatment}

All the patients received first-line treatment with either sorafenib or sunitinib as monotherapy. Sorafenib was administered at a dose of $400 \mathrm{mg}$ twice daily and sunitinib at a dose of $50 \mathrm{mg}$ q.d. Dose reduction or temporary suspension was carried out if grade 3-4 adverse event (AE) was reported according to the local prescribing information (PI). However, sorafenib dosage was increased to $600 \mathrm{mg}$ twice daily and subsequently to $800 \mathrm{mg}$ twice daily in some patients with disease progression.

\section{Outcomes and assessments}

The primary endpoint was OS (calculated from the date of first dose of sorafenib to the date of death or last follow-up) and PFS (time from first administration of 
sorafenib to the first documentation of disease progression or death from any cause). The effect of important prognostic factors such as age, gender, MSKCC score, ECOG performance and number of metastatic tumors on PFS and OS were evaluated.

\section{Statistical analysis}

Continuous variables such as PFS and OS were reported as medians and interquartile ranges, and categorical data such as age, gender, previous nephrectomy or systemic therapy were presented as proportions. The follow-up duration was calculated using reversed Kaplan-Meier method. The Shapiro-Wilk test was used to evaluate the data for normality distribution. OS and PFS were estimated using the Kaplan-Meier method with Rothman's 95\% CI and compared across groups using the log-rank test. The Cox proportional hazards model was used to evaluate the prognostic value of investigated parameters. All $p$ values were two-sided and were considered significant if <.05. The concordance index and the proportion of 2 explained variance ( $R$ ) was computed to assess the prediction performance for survival (PFS and OS). The statistical analysis of the collected data was performed using SPSS software version 19.

\section{Results}

\section{Patients and baseline demographics}

The data of 845 patients with mRCC enrolled between September 2006 and December 2014 were analyzed. Baseline demographics and clinical characteristics of the study population are presented in Table 1 . Of the 845 patients, 483 were treated with sorafenib and 362 were treated with sunitinib. Majority of patients were $\leq 65$ years of age and were predominantly men in both the treatment groups (age $\leq 65$ years: $77.2 \%$ vs. $85.4 \%$; males: $73.1 \%$ vs. $76.2 \%$ in sorafenib and sunitinib group, respectively). Approximately, $48 \%$ of the patients in the sorafenib group and $66 \%$ in the Sunitinib group had an ECOG performance status of 0 , and majority of patients in both the groups were at moderate risk according to the MSKCC score $(49.1 \%$ vs. $53 \%$ ) and Heng's score (47\% vs $51.7 \%$ ). There were significantly more number of patients with non-clear cell-type RCC in the sorafenib group (15.5\% vs. $8.6 \%$; $p=0.002$ ). However, the number of patients at low and moderate risk according to MSKCC score were significantly more in the sunitinib group and the number of patients at high risk according to MSKCC score were significantly more in the sorafenib group ( $15.1 \%$ vs. $5.2 \%$; $p<$ 0.001). No significant differences between the 2 treatment groups were observed for parameters such as gender, number of metastases, bone metastasis and simple bone metastasis.

\section{Endpoint analysis}

Survival data are presented in terms of OS and PFS. Median OS (mOS) and PFS (mPFS) for both the treatment groups are shown in Fig. 1. Median OS (months) for the sorafenib and sunitinib groups was found to be similar (24.0 vs. $24.0 ; p=0.298$ ). Overall, the sorafenib group exhibited higher mPFS (months) when compared to sunitinib group (11.1 vs. $10.0 ; p=0.028)$. Overall response rate $(\mathrm{CR}+\mathrm{PR})$ of sorafenib treatment was $16.77 \%(82 / 483)$ was lower than sunitinib treatment $20.99 \%$ (76/362). Disease control rate $(\mathrm{CR}+\mathrm{PR}+\mathrm{SD})$ was similar in 2 groups $88.61 \%$ (428/483) vs $88.39 \%$ (320/362). Disease progression was seen in $11.4 \%$ patients in sorafenib group and $11.6 \%$ in sunitinib. Only $27.3 \%$ in the sorafenib group and $22.4 \%$ in the sunitinib group had dose escalation which was considered as second line treatment.

\section{Predictor analysis}

Univariate analysis of 12 key including demographic and clinical characteristics identified pathology of RCC (clear cell and non-clear cell type), ECOG performance status, MSKCC score for risk, Heng's criteria for risk, number of metastasis, simple lung metastasis, bone metastasis and liver metastasis as significant predictors for OS $(p=$ $0.000)$ and PFS $(p=0.000)$. Additionally, simple bone metastasis was also identified as a significant predictor of PFS $(p=0.016)$. Data from univariate analysis of OS and PFS predictors are detailed in Table 2. However, multivariate analysis identified variables such as treatment (sorafenib vs. sunitinib), pathology, ECOG performance status, MSKCC scores, Heng's criteria of risk and number of metastases as significant predictors for OS (Fig. 2). For PFS, liver metastasis along with other variables reported for OS were identified as significant predictors (Table 3, Fig. 3). Clinical outcomes in terms of mOS seemed to be significantly better with sorafenib in patients older than 65 years $(p=0.041)$, ECOG of $0(p<0.001)$ and median MSKCC risk score $(p=0.008)$.

Further, multivariate analysis revealed significant association between OS and several predictors such as sorafenib treatment (HR 1.3, 95\% CI 1.096, 1.542; $p=0.003$ ), Clear cell type RCC (HR 1.49, 95\% CI 1.167, 1.922; $p=0.002)$, ECOG grade 3 (HR 1.22, 95\% CI 1.082, 1.385; $p=0.001$ ), high grade MSKCC 3 (HR 1.36, 95\% CI 1.086, 1.703; $p=0.007$ ), Heng risk (HR 1.53, 95\% CI 1.244, 1.889; $p<0.0001$ ), presence of liver (HR 1.40, 95\% CI 1.067, 1.846; $p=0.001$ ), and lymph node metastases (HR 1.22, 95\% CI 1.082, 1.385; $p=0.001$ ). Other prognostic factors such as gender, age, second line treatment, lung and bone metastasis and previous nephrectomy showed no significant association with OS (Table 4).

\section{Discussion}

Sorafenib and sunitinib have been used for mRCC in China since 2007. Since then several studies have tried 
Table 1 Baseline characteristics of the study population

\begin{tabular}{|c|c|c|c|c|}
\hline Clinical variable & & Sorafenib, n (\%) & Sunitinib, n (\%) & $P$ \\
\hline \multirow[t]{2}{*}{ Age (years) } & $<65$ & $373(77.2)$ & $309(85.4)$ & 0.004 \\
\hline & $\geq 65$ & $110(22.8)$ & $53(14.6)$ & \\
\hline \multirow[t]{2}{*}{ Gender } & Male & $353(73.1)$ & $276(76.2)$ & 0.302 \\
\hline & Female & $130(26.9)$ & $86(23.8)$ & \\
\hline \multirow[t]{2}{*}{ Pathology } & Clear cell type & $408(84.5)$ & $331(91.4)$ & 0.002 \\
\hline & Non-clear cell type & $75(15.5)$ & $31(8.6)$ & \\
\hline \multirow[t]{4}{*}{ ECOG } & 0 & $230(47.6)$ & $238(65.7)$ & $<0.001$ \\
\hline & 1 & $188(38.9)$ & $94(26.0)$ & \\
\hline & 2 & $58(12.0)$ & $29(8.0)$ & \\
\hline & 3 & $7(1.4)$ & $1(0.3)$ & \\
\hline \multirow[t]{2}{*}{ Previous nephrectomy } & Yes & $376(77.8)$ & $298(82.3)$ & 0.120 \\
\hline & No & $107(22.2)$ & $64(17.7)$ & \\
\hline \multirow[t]{3}{*}{ MSKCC } & Low risk & $173(35.8)$ & $151(41.7)$ & $<0.001$ \\
\hline & Moderate risk & $237(49.1)$ & $192(53.0)$ & \\
\hline & High risk & $73(15.1)$ & $19(5.2)$ & \\
\hline \multirow[t]{3}{*}{ Heng's criteria } & Low risk & $183(37.9)$ & $144(39.8)$ & 0.016 \\
\hline & Moderate risk & $227(47.0)$ & $187(51.7)$ & \\
\hline & High risk & $73(15.1)$ & $31(8.6)$ & \\
\hline \multirow[t]{4}{*}{ Number of metastatic organs } & 1 & $207(42.9)$ & $174(48.1)$ & 0.129 \\
\hline & 2 & $182(37.7)$ & $109(30.1)$ & \\
\hline & 3 & $75(15.5)$ & $66(18.2)$ & \\
\hline & 4 & $19(3.9)$ & $13(3.6)$ & \\
\hline \multirow[t]{2}{*}{ Lung metastasis } & No & $174(36.0)$ & $106(29.3)$ & 0.046 \\
\hline & Yes & $309(64.0)$ & $256(70.7)$ & \\
\hline \multirow[t]{2}{*}{ Simple lung metastasis } & No & $380(78.7)$ & $251(69.3)$ & 0.002 \\
\hline & Yes & $103(21.3)$ & $111(30.7)$ & \\
\hline \multirow[t]{2}{*}{ Bone metastasis } & No & $319(66.0)$ & $254(70.2)$ & 0.207 \\
\hline & Yes & $164(34.0)$ & $108(29.8)$ & \\
\hline \multirow[t]{2}{*}{ Simple bone metastasis } & No & $445(92.1)$ & $341(94.2)$ & 0.276 \\
\hline & Yes & $38(7.9)$ & $21(5.8)$ & \\
\hline \multirow[t]{2}{*}{ Liver metastasis } & No & $421(87.2)$ & $335(92.5)$ & 0.013 \\
\hline & Yes & $62(12.8)$ & $27(7.5)$ & \\
\hline \multirow[t]{2}{*}{ Lymph node metastasis } & No & $323(66.9)$ & $248(68.5)$ & 0.656 \\
\hline & Yes & $260(33.1)$ & $114(31.5)$ & \\
\hline \multirow[t]{4}{*}{ RECIST response } & $C R$ & $5(1.0)$ & $4(1.1)$ & \\
\hline & $P R$ & $77(15.9)$ & $72(19.9)$ & \\
\hline & SD & $346(71.6)$ & $244(67.4)$ & \\
\hline & PD & $55(11.4)$ & 42 (11.6) & 0.110 \\
\hline \multirow[t]{2}{*}{ Second line treatment } & Yes & $132(27.3)$ & $81(22.4)$ & \\
\hline & No & $351(72.7)$ & $281(77.6)$ & \\
\hline
\end{tabular}

ECOG eastern cooperative oncology group, MSKCC memorial sloan-kettering cancer centre, OS overall survival, $P F S$ progression-free survival

to elucidate the efficacy of the novel treatments. OS is a reliable endpoint for assessing the efficacy of $\mathrm{mRCC}$ with targeted therapy [21]. Although, these new therapies have improved the OS and PFS of patients with $\mathrm{mRCC}$, gradual development of drug resistance may often lead to switching one therapy to other, resulting in sequential 
Survival Function
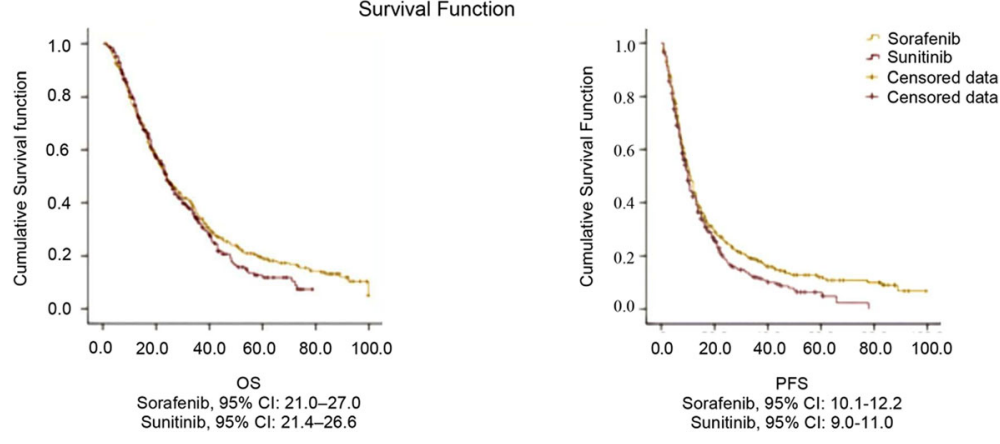

Fig. 1 Survival data of first-line targeted therapy for advanced renal carcinoma

therapy. Filson et al. reported that patients on first-line sorafenib therapy have high probability of proceeding to second-line therapy with sunitinib [22]. This clearly indicates the difference between the efficacies of the two drugs. However, a Korean study revealed comparable efficacy outcomes of sorafenib and sunitinib when used as monotherapy in first-line settings [20]. In support to the Korean study, more Asian studies are warranted to elucidate the efficacies of these therapies to inform the choice of first-line therapies.

Evidence-based studies suggest both first- and secondline efficacy of sorafenib. Earlier trials have shown comparable efficacy of sorafenib with interferon alpha-2a (PFS: 5.7 vs 5.6 months respectively), were the sorafenib treatment was well tolerated by the patients [23]. Also, in the INTORSECT trial, sorafenib had significantly longer OS compared to temsirolimus in first line therapy [7]. Further, a non-randomized open access trial demonstrated that sorafenib provides similar benefits in both first- and second-line setting [15]. The recent findings from an Italian study further confirmed that sorafenib prolonged PFS and OS in both first- and second-line routine community practice setting [24]. On the other hand, sunitinib also has established efficacy in previous phase 3 trials. Further, the efficacy of sunitinib was found to be superior to IFN-alpha but comparable to pazopanib across the trails, however severe grade 3 or 4 adverse effects were the limitations $[8,25]$.

Though efficacies of both the drugs in first-line setting are well described in retrospective literature, head-tohead comparison of real world clinical outcomes in patients are more heterogeneous than those trailed under controlled conditions, were patients with independent prognostic risk factors such as elderly, ECOG performance status and MSKCC Moderate risk groups were excluded. Considering the limitation of the clinical trials, expanded-access studies were conducted in America [26] and Europe [27] on sorafenib and one study on sunitinib [28], which were close to the real world scenario. Further, patient age may be a pivotal prognostic factor as elderly patients tend to have lower ECOG performance status than younger patients [29]. Hence, head-to-head comparisons are needed to inform the choice of treatment for such selected patients.

Our previous report documented higher clinical benefit rate in sorafenib treated patients than sunitinib treated patients $(94.67 \%$ vs $84.33 \%)$ [30]. In extension to this, the present retrospective review reported similar effectiveness of sorafenib and sunitinib in treating Chinese patients with $\mathrm{mRCC}$, however, sorafenib therapy was more effective in elderly patients ( $\geq 65$ years) and in patients with an normal performance status who were classified under MSKCC moderate risk category. A sub analysis of elderly patients in a phase 3 trial revealed a significant PFS advantage of sorafenib regardless of age [19]. In contrast, expanded-access studies on the clinical outcomes of sunitinib reproduced consistent efficacy and safety outcomes with previous results, and the outcomes were fairly similar in both elderly and younger populations but with significantly more common adverse effects seen in older population. However the clinical benefits of OS and PFS were inferior compared with placebo [28]. In line with our findings, a Swedish register-based demonstrated no difference between sorafenib and sunitinib in the duration of treatment or time to death when used as first-line therapy, however, the impact of the duration of first-line treatment differed in sequential therapy, concluding sorafenib first line treatment as a favorable choice in mRCC [31]. Furthermore, comparison of the present mOS findings with sorafenib therapy with other Asian studies demonstrated mixed results, where the mOS was consistent with a Chinese study12 conducted by Yu et al. [32], but were lower than the other Chinese [33, 34] and Korean studies [20]. However, higher mPFS with sorafenib over sunitinib was demonstrated in this study compared to previous Chinese [32], Korean [20, 35] and Italian [24] studies. The discrepancy may be related to diversity of patient populations enrolled in each study differing in many aspects related to prognosis and ethnicity. Furthermore, studies suggest that compared with Western patients, 
Table 2 Univariate analysis of predictors for OS and PFS

\begin{tabular}{|c|c|c|c|c|c|}
\hline Clinical variable & & mOS (months) & Log Rank test, $P$ & mPFS (months) & Log Rank test, $P$ \\
\hline \multirow[t]{2}{*}{ Gender } & Male & 24.0 & 0.413 & 11.0 & 0.131 \\
\hline & Female & 23.0 & & 10.0 & \\
\hline \multirow[t]{2}{*}{ Age } & $<65$ years & 24.0 & 0.714 & 10.6 & 0.435 \\
\hline & $\geq 65$ years & 24.0 & & 11.0 & \\
\hline \multirow[t]{2}{*}{ Pathology } & Clear cell type & 25.5 & $<0.001$ & 11.3 & $<0.001$ \\
\hline & Non-clear cell type & 14.0 & & 7.0 & \\
\hline \multirow[t]{4}{*}{ ECOG } & 0 & 30.0 & $<0.001$ & 12.1 & $<0.001$ \\
\hline & 1 & 22.1 & & 10.6 & \\
\hline & 2 & 11.0 & & 5.9 & \\
\hline & 3 & 8.8 & & 3.4 & \\
\hline \multirow[t]{2}{*}{ Previous nephrectomy } & Yes & 26.7 & $<0.001$ & 11.6 & $<0.001$ \\
\hline & No & 14.0 & & 7.0 & \\
\hline \multirow[t]{3}{*}{ MSKCC } & Low risk & 39.0 & $<0.001$ & 15.0 & $<0.001$ \\
\hline & Moderate risk & 22.0 & & 9.5 & \\
\hline & High risk & 9.3 & & 5.2 & \\
\hline \multirow[t]{3}{*}{ HENG } & Low risk & 39.0 & $<0.001$ & 15.0 & $<0.001$ \\
\hline & Moderate risk & 22.0 & & 9.4 & \\
\hline & High risk & 10.3 & & 5.8 & \\
\hline \multirow[t]{4}{*}{ Number of metastatic organs } & 1 & 32.0 & $<0.001$ & 14.0 & $<0.001$ \\
\hline & 2 & 21.0 & & 9.6 & \\
\hline & 3 & 15.3 & & 8.0 & \\
\hline & 4 & 16.0 & & 7.0 & \\
\hline \multirow[t]{2}{*}{ Lung metastasis } & No & 23.0 & 0.362 & 10.0 & 0.429 \\
\hline & Yes & 24.0 & & 11.0 & \\
\hline \multirow[t]{2}{*}{ Simple lung metastasis } & No & 21.4 & $<0.001$ & 9.3 & $<0.001$ \\
\hline & Yes & 32.4 & & 15.0 & \\
\hline \multirow[t]{2}{*}{ Bone metastasis } & No & 26.0 & $<0.001$ & 12.0 & 0.001 \\
\hline & Yes & 20.4 & & 9.0 & \\
\hline \multirow[t]{2}{*}{ Simple bone metastasis } & No & 24.0 & 0.182 & 10.5 & 0.016 \\
\hline & Yes & 24.0 & & 12.0 & \\
\hline \multirow[t]{2}{*}{ Liver metastasis } & No & 25.0 & $<0.001$ & 11.3 & $<0.001$ \\
\hline & Yes & 15.0 & & 6.0 & \\
\hline \multirow[t]{2}{*}{ Lymph node metastasis } & No & 27.5 & $<0.001$ & 12.0 & $<0.001$ \\
\hline & Yes & 18.0 & & 9.0 & \\
\hline \multirow[t]{4}{*}{ RECIST response } & $C R$ & 60.0 & $<0.001$ & 31.5 & $<0.001$ \\
\hline & $P R$ & 36.0 & & 20.7 & \\
\hline & SD & 23.3 & & 10.4 & \\
\hline & PD & 8.3 & & 3.0 & \\
\hline \multirow[t]{2}{*}{ Second line treatment } & Yes & 30.0 & 0.024 & 10.9 & 0.363 \\
\hline & No & 22.0 & & 10.5 & \\
\hline
\end{tabular}

ECOG eastern cooperative oncology group, MSKCC memorial sloan-kettering cancer centre, OS overall survival, PFS progression-free survival

Chinese patients respond better to sorafenib as first-line targeted therapy [36, 37] Also, the results from TIVO-1 trial suggested that sorafenib as a first-line mRCC therapy yielded PFS of 9.1 month [38], which was lesser than the findings from the present study (PFS 11.1 months). Hence, the present study findings further support the previous 


\begin{tabular}{|c|c|c|c|}
\hline & & $\mathrm{HR}(95 \% \mathrm{CI})$ & $\mathrm{p}$ value \\
\hline Drug:Sorafenib vs Sunitinib & $\longrightarrow$ & $1.283(1.08,1.524)$ & 0.005 \\
\hline Gender:Male vs Female & - & $1.002(0.834,1.204)$ & 0.984 \\
\hline Age $:<65$ vs $>=65$ & - & $0.971(0.789,1.196)$ & 0.784 \\
\hline Pathology:Clear cell vs Non clear cell & & $1.498(1.167,1.924)$ & 0.002 \\
\hline ECOG:0 vs 1 vs 2 vs 3 & $\rightarrow$ & $1.219(1.078,1.379)$ & 0.002 \\
\hline Previous nephrectomy:No vs Yes & & $0.872(0.697,1.09)$ & 0.229 \\
\hline MSKCC:Low vs median vs High & | & $1.354(1.081,1.694)$ & 0.008 \\
\hline HENG:Low vs median vs High & & $1.526(1.239,1.88)$ & $<0.0001$ \\
\hline Number of metastatic organs: 1 vs 2 vs 3 vs 4 & $\rightarrow$ & $1.149(0.982,1.345)$ & 0.084 \\
\hline Lung metastasis:Yes vs No & & $1.285(1.005,1.642)$ & 0.045 \\
\hline Single hung metastasis:No vs Yes & & $0.831(0.624,1.106)$ & 0.204 \\
\hline Bone metastasis:Yes vs No & & $1.002(0.798,1.258)$ & 0.985 \\
\hline Single bone metastasis:No vs Yes & & $0.972(0.634,1.492)$ & 0.897 \\
\hline Liver metastasis:Yes vs No & & $1.398(1.062,1.84)$ & 0.017 \\
\hline Lymph node metastasis:Yes vs No & $\rightarrow$ & $1.289(1.054,1.577)$ & 0.014 \\
\hline Second line treatment:No vs Yes & & $0.886(0.737,1.065)$ & 0.198 \\
\hline & & & \\
\hline
\end{tabular}

Fig. 2 Multivariate analysis of predictors of OS. ECOG, Eastern Cooperative Oncology Group; MSKCC, Memorial Sloan-Kettering Cancer Centre; OS, overall survival

findings with regards to superior efficacy of sorafenib in Chinese patients with mRCC.

Furthermore, multivariate regression analysis revealed treatment (Sorafenib vs. Sunitinib), pathology, ECOG performance status, MSKCC scores, HENG criteria of risk, and number of metastases as significant predictors for OS which is in line with the previous studies conducted by Motzar et al., which demonstrated ethnicity, ECOG status, bone metastasis and old age as predictors for OS and PFS in first-line therapy with sunitinib [39]. The findings are also consistent with the findings of
Yang et al. who reported MSKCC status as prognostic factor for OS and PFS when treated with sunitinib [40]. Overall, our study findings support the findings of the Swedish and Italian studies, demonstrating comparable efficacy of sorafenib over sunitinib, but a more favorable sorafenib therapy in elderly and moderate risk mRCC patients.

Our study has several limitations. The retrospective design of the study comes as an inherent limitation; however, relatively large sample size compared to previous retrospective studies may power the study. The

Table 3 Multivariate analysis of predictors for PFS

\begin{tabular}{|c|c|c|c|c|c|}
\hline \multirow[t]{2}{*}{ Variables } & & \multirow[t]{2}{*}{$P$} & \multirow[t]{2}{*}{$\mathrm{HR}$} & \multicolumn{2}{|c|}{$95.0 \% \mathrm{Cl}$} \\
\hline & & & & Lower & Upper \\
\hline Drug & Sorafenib vs Sunitinib & $<0.001$ & 1.420 & 1.211 & 1.664 \\
\hline Gender & Male vs Female & 0.414 & 1.074 & 0.906 & 1.273 \\
\hline Age & $<65$ vs $>=65$ & 0.194 & 0.879 & 0.723 & 1.068 \\
\hline Pathology & Clear cell vs Non clear cell & 0.024 & 1.312 & 1.037 & 1.660 \\
\hline ECOG & 0 vs 1 vs 2 vs 3 & $<0.001$ & 1.245 & 1.105 & 1.402 \\
\hline Previous nephrectomy & No vs Yes & 0.500 & 0.931 & 0.755 & 1.147 \\
\hline MSKCC & Low vs median vs High & 0.043 & 1.253 & 1.007 & 1.558 \\
\hline HENG & Low vs median vs High & $<0.001$ & 1.458 & 1.198 & 1.774 \\
\hline Number of metastatic organs & 1 vs 2 vs 3 vs 4 & 0.679 & 1.033 & 0.886 & 1.204 \\
\hline Lung metastasis & Yes vs No & 0.175 & 1.173 & 0.931 & 1.478 \\
\hline Single lung metastasis & No vs Yes & 0.188 & 0.834 & 0.637 & 1.092 \\
\hline Bone metastasis & Yes vs No & 0.108 & 1.193 & 0.962 & 1.481 \\
\hline Single bone metastasis & No vs Yes & 0.085 & 0.703 & 0.470 & 1.050 \\
\hline Liver metastasis & Yes vs No & $<0.001$ & 1.645 & 1.263 & 2.142 \\
\hline Lymph node metastasis & Yes vs No & 0.023 & 1.250 & 1.031 & 1.516 \\
\hline Second line treatment & No vs Yes & $<0.001$ & 1.398 & 1.184 & 1.651 \\
\hline
\end{tabular}




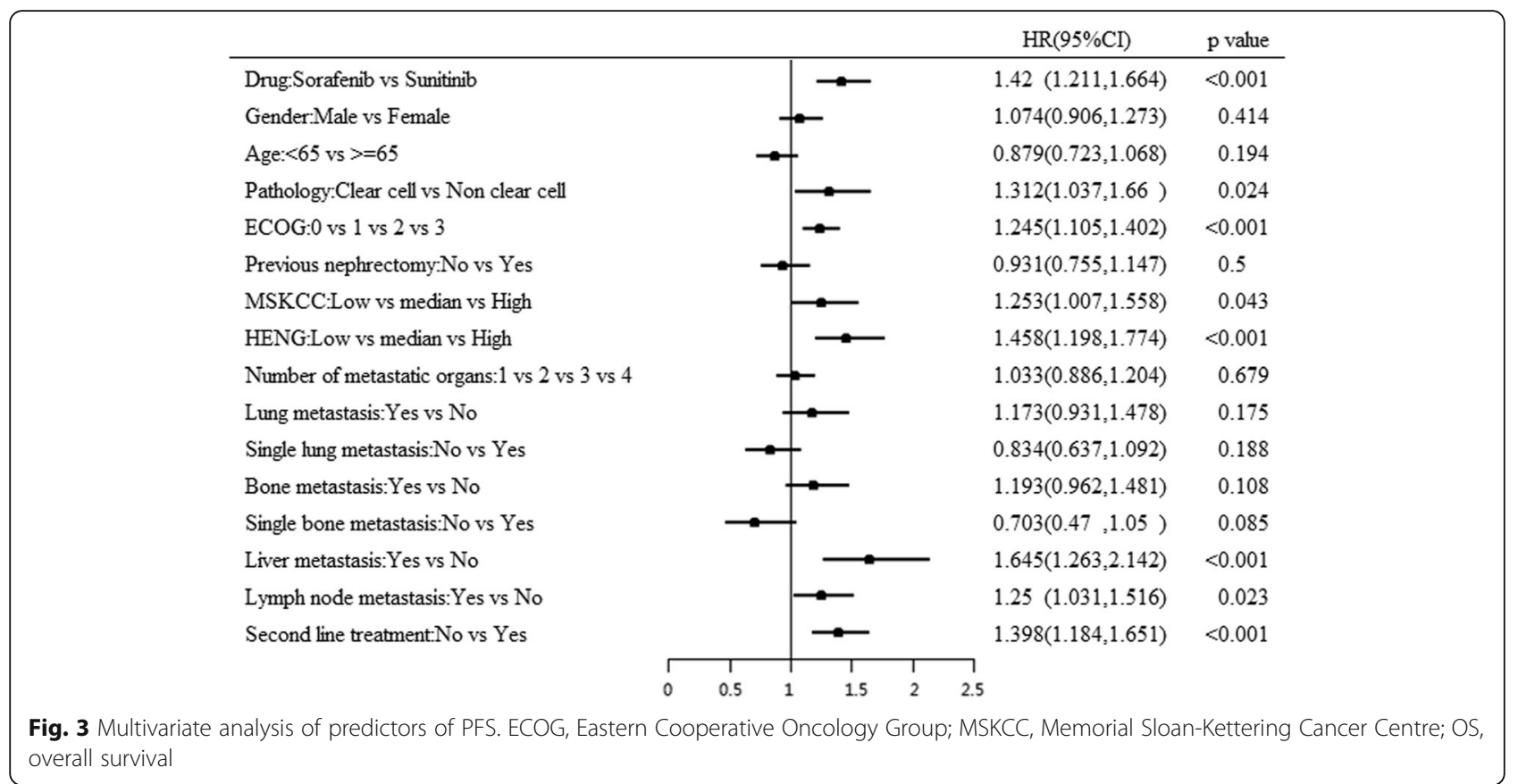

favorable outcomes of sorafenib demonstrated in this study may create a conflict in the belief of the clinicians who believe sunitinib as a better first-line option. Further, mOS was chosen as an endpoint and hence, the survival probability at a later time point after treatment initiation could not be established, hence, warranting further long term comparative efficacy studies.

\section{Conclusion}

The present study suggests that sorafenib and sunitinib are both effective as first-line therapeutic agents in treating Chinese patients with mRCC. Sorafenib is effective in elderly patients ( $\geq 65$ years) and in patients with an ECOG status of 0 , classified under MSKCC moderate risk. In addition, multivariate analysis suggests that variables such

Table 4 Multivariate analysis of predictors for OS

\begin{tabular}{|c|c|c|c|c|c|}
\hline \multirow[t]{2}{*}{ Variables } & & \multirow[t]{2}{*}{$P$} & \multirow[t]{2}{*}{$\mathrm{HR}$} & \multicolumn{2}{|c|}{$95.0 \% \mathrm{Cl}$} \\
\hline & & & & Lower & Upper \\
\hline Drug & Sorafenib vs Sunitinib & 0.005 & 1.283 & 1.080 & 1.524 \\
\hline Gender & Male vs Female & 0.984 & 1.002 & 0.834 & 1.204 \\
\hline Age & $<65$ vs $>=65$ & 0.784 & 0.971 & 0.789 & 1.196 \\
\hline Pathology & Clear cell vs Non clear cell & 0.002 & 1.498 & 1.167 & 1.924 \\
\hline ECOG & 0 vs 1 vs 2 vs 3 & 0.002 & 1.219 & 1.078 & 1.379 \\
\hline Previous nephrectomy & No vs Yes & 0.229 & 0.872 & 0.697 & 1.090 \\
\hline MSKCC & Low vs median vs High & 0.008 & 1.354 & 1.081 & 1.694 \\
\hline HENG & Low vs median vs High & $<0.0001$ & 1.526 & 1.239 & 1.880 \\
\hline Number of metastatic organs & 1 vs 2 vs 3 vs 4 & 0.084 & 1.149 & 0.982 & 1.345 \\
\hline Lung metastasis & Yes vs No & 0.045 & 1.285 & 1.005 & 1.642 \\
\hline Single lung metastasis & No vs Yes & 0.204 & 0.831 & 0.624 & 1.106 \\
\hline Bone metastasis & Yes vs No & 0.985 & 1.002 & 0.798 & 1.258 \\
\hline Single bone metastasis & No vs Yes & 0.897 & 0.972 & 0.634 & 1.492 \\
\hline Liver metastasis & Yes vs No & 0.017 & 1.398 & 1.062 & 1.840 \\
\hline Lymph node metastasis & Yes vs No & 0.014 & 1.289 & 1.054 & 1.577 \\
\hline Second line treatment & No vs Yes & 0.198 & 0.886 & 0.737 & 1.065 \\
\hline
\end{tabular}


as treatment (sorafenib vs sunitinib), pathology, ECOG performance status, MSKCC scores, Heng's criteria of risk and number of metastases are significant prognostic factors for OS and PFS.

\section{Abbreviations}

AE: Adverse effect; CT: Computer tomography; DFS: Disease free survival; ECOCG: Eastern cooperative oncology group performance status; mOS: Median overall survival; mPFS: Median progression free survival; mRCC: Metastatic renal cell carcinoma; MRI: Magnetic resonance imaging; MSKCC: Memorial sloan-kettering cancer centre; ORR: Objective response rate; OS: Overall survival; PFS: Progression free survival; PI: Prescribing information; QoL: Quality of life; RECIST: Response evaluation criteria in solid tumors

\section{Acknowledgements}

Not applicable

\section{Funding}

This work was partially supported by grants from the Shanghai leaders' foundation, project 2014ZYJB0102 supported by the Shanghai Municipal Commission of Health and Family Planning grant and The Project of the National Natural Science Foundation of China (Grant No. 81001131, 81370073 and 81202004). The funders had no role to play in the design of the study and collection, analysis, and interpretation of data and in writing the manuscript.

\section{Availability of data and materials}

The data used in this study are maintained at the Beijing Cancer Hospital Fudan University Shanghai Cancer Centre and the Peking University First Hospital; however they cannot be made available as they contain individual level health information

\section{Authors' contributions}

HLZ and XNS were responsible for study design, planning the analysis, analyzing and interpretation of data. JG and DWY were responsible for analysis, and interpretation of the data and data revisions and writing the manuscript. XL, HKW, ZHC and ZSH gave input at all stages of the analysis. All the authors have read and approved the final version of the manuscript

\section{Competing interests}

The authors declare that they have no competing interests.

\section{Consent to publish}

Not applicable.

\section{Ethics approval and consent to participate}

Ethical approval was obtained from institutional ethics committee of Beijing Cancer Hospital, Fudan University Shanghai Cancer Centre and the Peking University First Hospital. This study was conducted in accordance with the Declaration of Helsinki and its subsequent revisions. Signed informed consent was obtained from the patients.

\section{Author details}

'Department of Urology, Fudan University Shanghai Cancer Center, Shanghai 200032, People's Republic of China. ${ }^{2}$ Department of Oncology, Shanghai Medical College, Fudan University, Shanghai 200032, People's Republic of China. ${ }^{3}$ Key Laboratory of Carcinogenesis and Translational Research (Ministry of Education/Beijing), Department of Renal Cancer and Melanoma, Peking University Cancer Hospital \& Institute, Beijing, People's Republic of China. ${ }^{4}$ Department of Urology, Peking University First Hospital, Institute of Urology, National Urological Cancer Center, Peking University, Beijing, People's Republic of China.

Received: 16 July 2016 Accepted: 16 December 2016 Published online: 05 January 2017

\section{References}

1. Wersall PJ, Blomgren H, Lax I, et al. Extracranial stereotactic radiotherapy for primary and metastatic renal cell carcinoma. Radiother Oncol. 2005;77(1):88-95.
2. Siegel RL, Miller KD, Jemal A. Cancer statistics, 2015. CA Cancer J Clin. 2015;65(1):5-29.

3. Motzer RJ, Basch E. Targeted drugs for metastatic renal cell carcinoma. Lancet. 2007:370(9605):2071-3.

4. Escudier B, Pluzanska A, Koralewski P, et al. AVOREN Trial investigators. Bevacizumab plus interferon alfa-2a for treatment of metastatic renal cell carcinoma: a randomised, double-blind phase III trial. Lancet. 2007:370(9605):2103-11.

5. Groupe Français d'Immunothérapie, Negrier S, Escudier B, Lasset C, et al. Recombinant human interleukin-2, recombinant human interferon alfa-2a, or both in metastatic renal-cell carcinoma. N Engl J Med. 1998;338(18):1272-8.

6. Escudier B, Desai AA, Rolland F, et al. Sorafenib in advanced clear-cell renalcell carcinoma. N Engl J Med. 2007;356:125-34.

7. Hutson TE, Escudier B, Esteban E, et al. Randomized phase III trial of temsirolimus versus sorafenib as second-line therapy after sunitinib in patients with metastatic renal cell carcinoma. J Clin Oncol. 2014;32(8):760-7.

8. Motzer RJ, Hutson TE, Tomczak P, et al. Sunitinib versus interferon alfa in metastatic renal-cell carcinoma. Engl J Med. 2007;356(2):115-24

9. Sternberg CN, Hawkins RE, Wagstaff J, et al. A randomised, double-blind phase III study of pazopanib in patients with advanced and/or metastatic renal cell carcinoma: final overall survival results and safety update. Eur J Cancer. 2013;49(6):1287-96.

10. Motzer RJ, Escudier B, Oudard S, et al. RECORD-1 Study Group. Phase 3 trial of everolimus for metastatic renal cell carcinoma: final results and analysis of prognostic factors. Cancer. 2010;116(18):4256-65.

11. Motzer RJ, Escudier B, Tomczak P, et al. Axitinib versus sorafenib as secondline treatment for advanced renal cell carcinoma: overall survival analysis and updated results from a randomised phase 3 trial. Lancet Oncol. 2013;14(6):552-62.

12. Scher HI, Halabi S, Tannock I, et al. Design and end points of clinical trials for patients with progressive prostate cancer and castrate levels of testosterone: recommendations of the Prostate Cancer Clinical Trials Working Group. J Clin Oncol. 2008;26:1148-59.

13. Wilson MK, Karakasis K, Oza AM. Outcomes and endpoints in trials of cancer treatment: the past, present, and future. Lancet Oncol. 2015;16:e32-42.

14. Pazdur R. Endpoints for assessing drug activity in clinical trials. Oncologist. 2008;13:19-21

15. Hutson TE, Lesovoy V, Al-Shukri S, et al. Axitinib versus sorafenib as first-line therapy in patients with metastatic renal-cell carcinoma: a randomised open-label phase 3 trial. Lancet Oncol. 2013;14(13):1287-94.

16. Motzer RJ, Hutson TE, Cella D, et al. Pazopanib versus sunitinib in metastatic renal-cell carcinoma. Engl J Med. 2013;369(8):722-31.

17. Sherman S, Amzal B, Calvo E, et al. An Indirect Comparison of Everolimus Versus Axitinib in US Patients With Advanced Renal Cell Carcinoma in Whom Prior Sunitinib Therapy Failed. Clin Ther. 2015.

18. Zanardi E, Verzoni $E$, Grassi P, et al. Clinical experience with temsirolimus in the treatment of advanced renal cell carcinoma. Ther Adv Urol. 2015:7(3):152-61.

19. Sheng $X$, Chi Z, Cui $C$, et al. Efficacy and safety of sorafenib versus sunitinib as first-line treatment in patients with metastatic renal cell carcinoma: largest single-center retrospective analysis. Oncotarget. 2016;10;7(19):27044-54.

20. Park SJ, Lee JL, Park I, et al. Comparative efficacy of sunitinib versus sorafenib as first-line treatment for patients with metastatic renal cell carcinoma. Chemotherapy. 2012:58(6):468-74.

21. Zhao J, Huang $X$, Sun $F$, et al. Prognostic factors for overall survival with targeted therapy in Chinese patients with metastatic renal cell carcinoma. Can Urol Assoc J. 2014;8(11-12):E821-7.

22. Filson CP, Redman BG, Dunn RL, Miller DC. Initial patterns of care with oral targeted therapies for patients with renal cell carcinoma. Urology. 2011;77:825-30

23. Escudier B, Szczylik C, Hutson TE, et al. Randomized Phase II trial of first-line treatment with sorafenib versus interferon alfa-2a in patients with metastatic renal cell carcinoma. J Clin Oncol. 2009;27(8):1280-9.

24. Procopio G, Derosa L, Gernone A, et al. Sorafenib as first- or second-line therapy in patients with metastatic renal cell carcinoma in a community setting. Future Oncol. 2014;10:1741-50.

25. McCann L, Amit O, Pandite L, Amado RG. An indirect comparison analysis of pazopanib versus other agents in metastatic renal cell carcinoma (mRCC). J Clin Oncol. 2010;28(Suppl):e15128.

26. Stadler WM, Figlin RA, McDermott DF, et al. Safety and efficacy results of the advanced renal cell carcinoma sorafenib expanded access program in North America. Cancer. 2010;116(5):1272-80. 
27. Beck J, Procopio G, Bajetta E, et al. Final results of the European Advanced Renal Cell Carcinoma Sorafenib (EU-ARCCS) expanded-access study: a large open-label study in diverse community settings. Ann Oncol. 2011;22(8):1812-23.

28. Gore ME, Szczylik C, Porta C, et al. Final results from the large sunitinib global expanded-access trial in metastatic renal cell carcinoma. $\mathrm{Br} J$ Cancer. 2015;113(1):12-9.

29. Bellmunt J, Fishman M, Eisen T, Quinn D. Expert opinion on the use of firstline sorafenib in selected metastatic renal cell carcinoma patients. Expert Rev Anticancer Ther. 2010;10(6):825-35.

30. Eisen T, Oudard S, Szczylik C, et al. Sorafenib for older patients with renal cell carcinoma: subset analysis from a randomized trial. J Natl Cancer Inst. 2008;100(20):1454-63.

31. Ambring A, Björholt I, Lesen E, Stierner U, Oden A. Treatment with sorafenib and sunitinib in renal cell cancer: a Swedish register-based study. Med Oncol. 2013;30(1):331.

32. Yu X, Guo G, Li X, et al. Retrospective analysis of the efficacy and safety of sorafenib in Chinese patients with metastatic renal cell carcinoma and prognostic factors related to overall survival. Medicine (Baltimore). 2015;94:e1361.

33. Liu F, Chen X, Peng E, et al. VEGF pathway-targeted therapy for advanced renal cell carcinoma: a meta-analysis of randomized controlled trials. J Huazhong Univ Sci Technol Med Sci. 2011;31:799-806.

34. Guo F, Han T, Liu Z, et al. Prognostic analysis of Chinese patients with metastasis renal cell cancer receiving sorafenib: results from a multicentre long-term follow-up retrospective study. Onco Targets Ther. 2015;8:1581-8.

35. Kim SH, Kim S, Nam BH, et al. Efficacy and safety of sorafenib therapy on metastatic renal cell carcinoma in Korean patients: results from a retrospective multicentre study. PLoS One. 2015;10:e0135165.

36. Tan X, Liu Y, Hou J, Cao G. Targeted therapies for renal cell carcinoma in Chinese patients: focus on everolimus. Onco Targets Ther. 2015:8:313-21.

37. Ye DW, Zhang HL. Critical appraisal of sorafenib in the treatment of Chinese patients with renal cell carcinoma. Onco Targets Ther. 2014;7:925-35.

38. Motzer RJ, Nosov D, Eisen T, et al. Tivozanib versus sorafenib as initial targeted therapy for patients with metastatic renal cell carcinoma: results from a phase III trial. J Clin Oncol. 2013;31:3791-9.

39. Motzer RJ, Escudier B, Bukowski R, et al. Prognostic factors for survival in 1059 patients treated with sunitinib for metastatic renal cell carcinoma. Br J Cancer. 2013;108:2470-7.

40. Yang L, Shi L, Fu Q, Xiong H, Zhang M, Yu S. Efficacy and safety of sorafenib in advanced renal cell carcinoma patients: Results from a long-term study. Oncol Lett. 2012;3:935-9.

\section{Submit your next manuscript to BioMed Central and we will help you at every step:}

- We accept pre-submission inquiries

- Our selector tool helps you to find the most relevant journal

- We provide round the clock customer support

- Convenient online submission

- Thorough peer review

- Inclusion in PubMed and all major indexing services

- Maximum visibility for your research

Submit your manuscript at www.biomedcentral.com/submit 